\title{
ARTICLE
}

\section{Attempt at the Change of Decay Rate of Gold-198 by Using Weak-Interaction Field in Electrochemical Neutrino Detector}

\author{
Norichika TERAO $^{1 *}$, Hidehiko ARIMA ${ }^{1}$, Kenji ISHIBASHI $^{1}$, Shin $_{\text {ITO }}^{2}$, and Taeko KOGA ${ }^{2}$ \\ ${ }^{1}$ Department of Applied Quantum Physics and Nuclear Engineering, Kyushu University, 744, Motooka, Nishi-ku, Fukuoka 819-0395, Japan \\ ${ }^{2}$ Atomic Energy Research Institute, Kinki University, 3-4-1, Kowakae, Higashiosaka 577-8502, Japan
}

\begin{abstract}
An electrochemical apparatus was developed in our group for detecting neutrinos. The electrochemical detector was considered to make use of a biological product as an origin of weak-interaction through an axial-vector type scalar auxiliary field. The detector successfully responded to neutrinos. Some radioisotopes make beta decay to disintegrate into stable nuclei by weak-interaction. It is interesting to place a radioisotope sample inside the electrochemical detector and to observe whether the decay rate is influenced or not. As the radioisotope, activated gold of gold-198 was selected in this study. Gamma rays were measured by a germanium detector for an activated gold sample as reference data, while they were done for another activated gold sample which was placed in the electrochemical neutrino detector. The experiments were carried out with two detectors. The change of the half-life was unclear for these data. For evaluating the periodical variation of the beta decay rate, the measured counts were processed by Fast Fourier Transformation. A result showed that the component with periodicity of 6 hours gave statistically meaningful deviation ( $99 \%$ confidence) from the case of Poisson distribution. Further experiments may be required for studying reproduction of experimental results.
\end{abstract}

\section{KEYWORDS: electrochemical detector, neutrino, inner construction, scalar auxiliary field, decay rate}

\section{Introduction}

The radioisotopes (RIs) decay into other elements with a fixed half-life. It is quite difficult to change the half-life of RIs by the effect of external circumstantial conditions. In the case of electron capture (EC), however, it is possible to vary the half-life of RIs to a small extent by the change of physical environment, because the decay probability depends on the density of orbital electrons around the nucleus position. It was reported that the half-life of ${ }^{7} \mathrm{Be}$, which is one of low-atomic-number EC RIs, changes by about $0.15 \%$ by the effect of orbital electrons. ${ }^{1)}$

Neutrinos make the weak interaction, which is related to the beta decay. Although neither the weak interaction nor neutrino property is completely clarified yet, it is considered that an axial-vector type scalar auxiliary field $B^{0}$ takes an important role for both the weak interaction and the neutrino internal motion. ${ }^{2)}$ The scalar auxiliary field seemed to alter neutrino interaction. Once neutrinos go through the materials that produce the scalar auxiliary field, neutrinos may be fragmented into two groups of a weak-electric-charge-type constituent and weak-magnetic-moment-type ones due to the disagreement of kinetic mass with the weak-electromagnetic self-mass. ${ }^{3,4)}$

An electrochemical apparatus has been fabricated in our group for detecting neutrinos. ${ }^{3)}$ The electrochemical detector made use of raw silk as an origin of axial-vector type scalar auxiliary field $B^{0}$. The detector seemed to successfully respond to low energy neutrinos. The incident neutrinos were supposed to break into weak-charge type and magnetic-moment type fragments ${ }^{4}$, through the effect of scalar auxiliary field $B^{0}$. Beta-decay RIs are known to disintegrate into adjacent nuclei by weak-interaction. It is interesting to place a RI sample inside the electrochemical detector supposedly retaining $B^{0}$ and to observe whether the decay rate changes or not.

As the radioisotope, neutron-activated gold of ${ }^{198} \mathrm{Au}$ was selected $^{5)}$ in this study. Gamma rays were measured by a high-purity Ge detector both for an activated gold sample as reference data, and for another activated gold sample which was put in the water in the electrochemical neutrino detector. Deviation from the regular half-life was studied. A statistical analysis was made on periodical fluctuation of decaying counts.

\section{Experimental Apparatus}

We briefly explain the principle ${ }^{4)}$ of raw-silk type electrochemical detector shown in Fig. 1. The AV-type scalar auxiliary field $B^{0}$ is supposed to be generated around the raw silk. The field may disturb the mass generation mechanism of neutrinos, and break the neutrino into two fragments $\xi_{Q}$ and $\xi_{Q d}$. A water molecule receives the fragments and dissociates into $\mathrm{H}^{+} \xi_{Q}$ and $\mathrm{OH}^{-} \xi_{Q d}$. Signal generation scenario ${ }^{4)}$ is as follows.

Dissociation of water molecule:

$$
v+\mathrm{H}_{2} \mathrm{O} \rightarrow \mathrm{OH}^{-} \xi_{Q d}+\mathrm{H}^{+} \xi_{Q},
$$

Cathode reaction:

$$
2 \mathrm{OH}^{-} \xi_{Q d} \rightarrow \mathrm{H}_{2} \mathrm{O}+(1 / 2) \mathrm{O}_{2}+2 \mathrm{e}^{-} \xi_{Q d},
$$

Anode reaction:

$$
2 \mathrm{H}^{+} \xi_{Q}+(1 / 2) \mathrm{O}_{2}+2 \mathrm{e}^{-} \rightarrow \mathrm{H}_{2} \mathrm{O} \xi_{Q} .
$$

*Corresponding Author, E-mail:kenshin@meteor.nucl.kyushu-u.ac. jp 
Hydroxide ions with fragment $\xi_{Q d}$ move to the cathode, where water and oxygen molecules are generated as well as electrons with $\xi_{Q d}$. The oxygen molecules diffuse in the solution toward the anode. The electrons with $\xi_{\text {Od }}$ are conducted to the anode through copper wire. Meanwhile, the hydrogen ions with $\xi_{Q}$ diffuse to the anode, and they absorb the conducted electrons to form water molecules. Since $\xi_{Q d}$ produces an interaction energy with $B^{0}$, the difference of $B^{0}$ between two electrodes is supposed to finally make the voltage over the electrodes. The current is induced in the outer circuit of the detector.

\subsection{Structure of electrochemical detector}

The electrochemical detector is composed of Teflon container, gold plate, glassy carbon plate, raw silk, and purified water as shown in Fig. 1. The main part of the electrochemical detector was fabricated in the Teflon container with a size of $5.8 \mathrm{~cm}$ in diameter and $9.0 \mathrm{~cm}$ long. A gold and a glassy carbon plates were used as the cathode and anode, respectively. These plate sizes were both 20 $\mathrm{mm} \times 50 \mathrm{~mm}$ in surface, and the thickness were $0.1 \mathrm{~mm}$ (gold plate) and $1.0 \mathrm{~mm}$ (carbon plate), respectively. The raw silk of $0.5 \mathrm{~g}$ was set on the each side of the gold electrode and supposed to generate the AV-type scalar auxiliary field. Both plates worked as electrodes, and were connected to a voltage logger and a resistor that produced on input impedance of $1 \mathrm{M} \Omega$ thorough the copper conduction wires.

Purified water of $50 \mathrm{~g}$ was filled into the cylindrical Teflon container. The copper wires were connected to the gold and glassy carbon plates and these went across an O-ring to the outside of the Teflon container. The Teflon flange was attached to the Teflon container by using the O-ring and stainless-steel screws. The output voltage from the electrochemical detector was measured by using a data logger. The detector was placed in a temperature-controlled incubator and of which temperature was set at 27 degrees centigrade.

The output voltage of $50-60 \mathrm{mV}$ was usually obtained after operation of two weeks. When the detector was placed near the nuclear reactor, the voltage appreciably increases to twice value. Therefore, it was confirmed that the voltage was ascribed to incident neutrinos. For increasing the output voltage, raw silk of $100 \mathrm{~g}$ was immersed to purified water of $800 \mathrm{~g}$ and stored for a period above one month. Use of the 50 $\mathrm{g}$ water from the stored mixture leaded to the voltage exceeding $100 \mathrm{mV}^{4}$ )

\section{Beta-Decay Rate Experiment}

The nuclide ${ }^{198} \mathrm{Au}$ is a beta emitter, and decays into the isotope ${ }^{198} \mathrm{Hg}$ with a half-life of $2.695 \mathrm{~d}$. The RI ${ }^{198} \mathrm{Au}$ was produced in gold foil $(10 \mathrm{~mm} \times 10 \mathrm{~mm} \times 0.5 \mathrm{~mm})$ by ${ }^{197} \mathrm{Au}\left(\mathrm{n}_{\mathrm{th}}\right.$,

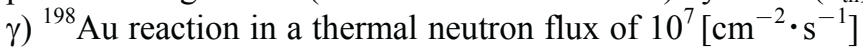
for 2 hours at Kinki University Atomic Energy Research Institute. Gamma rays were measured by a high-purity $\mathrm{Ge}$ detector for the activated gold sample in a black Teflon container as reference data. The area of photoelectric peak at

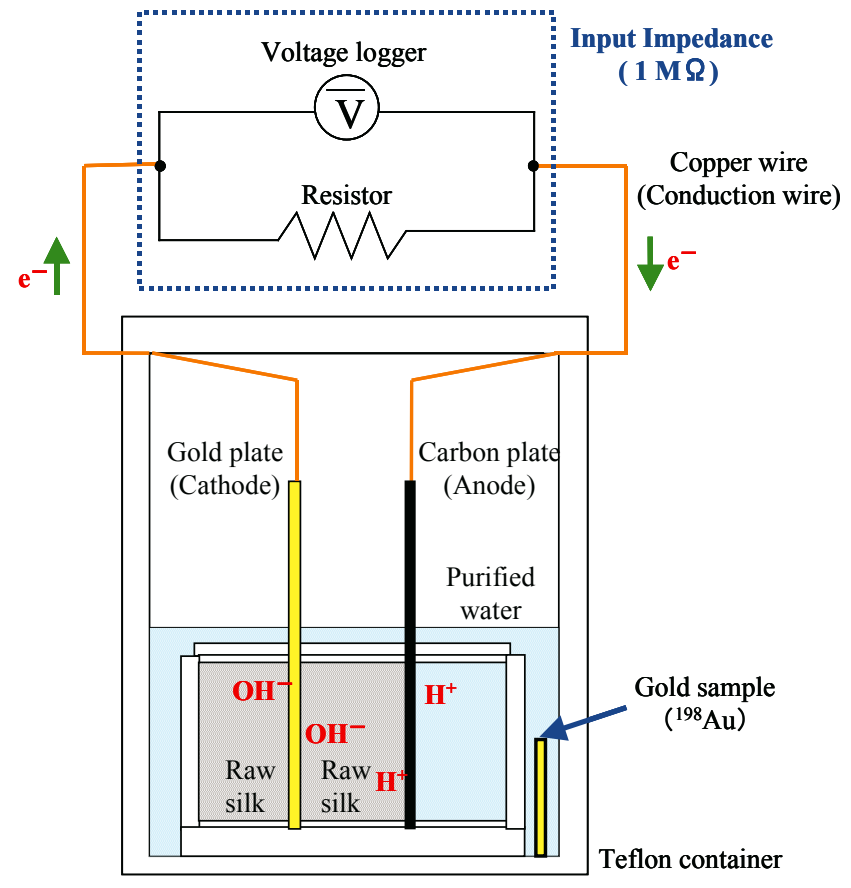

Fig. 1 Overview of the gold sample set in the electrochemical detector.

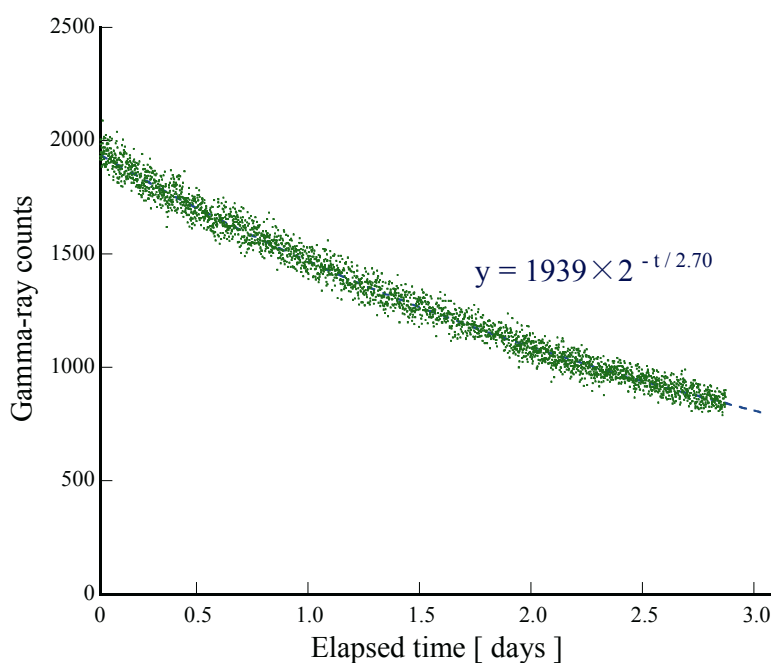

Fig. 2 Gamma ray counts of the $0.412-\mathrm{MeV}$ peak of gold sample set in a $135 \mathrm{mV}$ type detector (B). One fifth of total data are plotted. The dashed line indicates the least-squair-fit result.

$0.412-\mathrm{MeV}$ was counted. Then, another activated gold samples were put in the water of two individual electrochemical neutrino detectors. The gold sample is schematically illustrated in Fig. 1. Two electrochemical detectors were prepared that showed different output voltages: (A) one gave $60 \mathrm{mV}$ and (B) the other exhibited $135 \mathrm{mV}$. The gamma-ray measurement was continued for about three days.

The measured counts of $0.412-\mathrm{MeV}$ peak are shown in Fig. 2 for activated gold in detector (B). Each data point was obtained for a period of $15 \mathrm{~s}$ in measurement. All data were not plotted in this figure for ease of seeing. Other three 
Table 1 Half-lives of the $0.412-\mathrm{MeV}$ peaks getting from the approximation curve.

\begin{tabular}{c|c||cc}
\hline \multicolumn{2}{l||}{ Experiments } & Half-life & error \\
\hline \hline \multirow{2}{*}{$(1)$} & reference gold (a) & 2.718 & 0.006 \\
\cline { 2 - 2 } & $\begin{array}{c}\text { gold sample set in the } \\
\text { detector (A) }\end{array}$ & 2.712 & 0.008 \\
\hline \multirow{2}{*}{ (2) } & reference gold (b) & 2.676 & 0.006 \\
\cline { 2 - 2 } & $\begin{array}{c}\text { gold sample set in the } \\
\text { detector (B) }\end{array}$ & 2.698 & 0.006 \\
\hline
\end{tabular}

measurement data showed almost the same result as in Fig. 2. RThe values of half-life were listed in Table 1. The measured gamma-ray counts indicated that the half-life was unchanged. However, the decay rate seemed to have periodical fluctuation. The decay curve contains a certain random fluctuation, which is basically caused in the Poisson distribution in the decay events. We attempted to extract the periodical fluctuation from the experimental results.

\section{Detailed Analysis on Periodical Variation}

The periodical fluctuation is evaluated by the differences from the ideal Poisson distribution. The average of measured counts $\bar{x}$ is written by

$$
\bar{x}=\frac{1}{n} \sum_{i=1}^{n} x_{i}
$$

for experiments of $n$ times. The variance $\sigma^{2}$ is given by

$$
\sigma^{2}=\frac{1}{n} \sum_{i=1}^{n}\left(x_{i}-\bar{x}\right)^{2}
$$

This is rewritten as

$$
\sigma^{2}=\frac{1}{n} \sum_{i=1}^{n} x_{i}^{2}-(\bar{x})^{2}=\bar{x}^{2}-(\bar{x})^{2}
$$

The ratio of variance to mean value is defined as

$$
\frac{\sigma^{2}}{\bar{x}}=\frac{\overline{x^{2}}-(\bar{x})^{2}}{\bar{x}} \equiv 1+Y .
$$

The value $Y$ shows the correlativity of events. If the statistical data follows the Poisson distribution, $Y$ should be a value around zero. The each value of $Y$ was obtained by the use of 51 measurement data $(15 \times 51=765 \mathrm{~s}$ for a correlativity data point).

For evaluating the periodical variation of the beta decay rate, the values $Y$ were processed by Fast Fourier Transformation (FFT). FFT holds the symmetry of Discrete

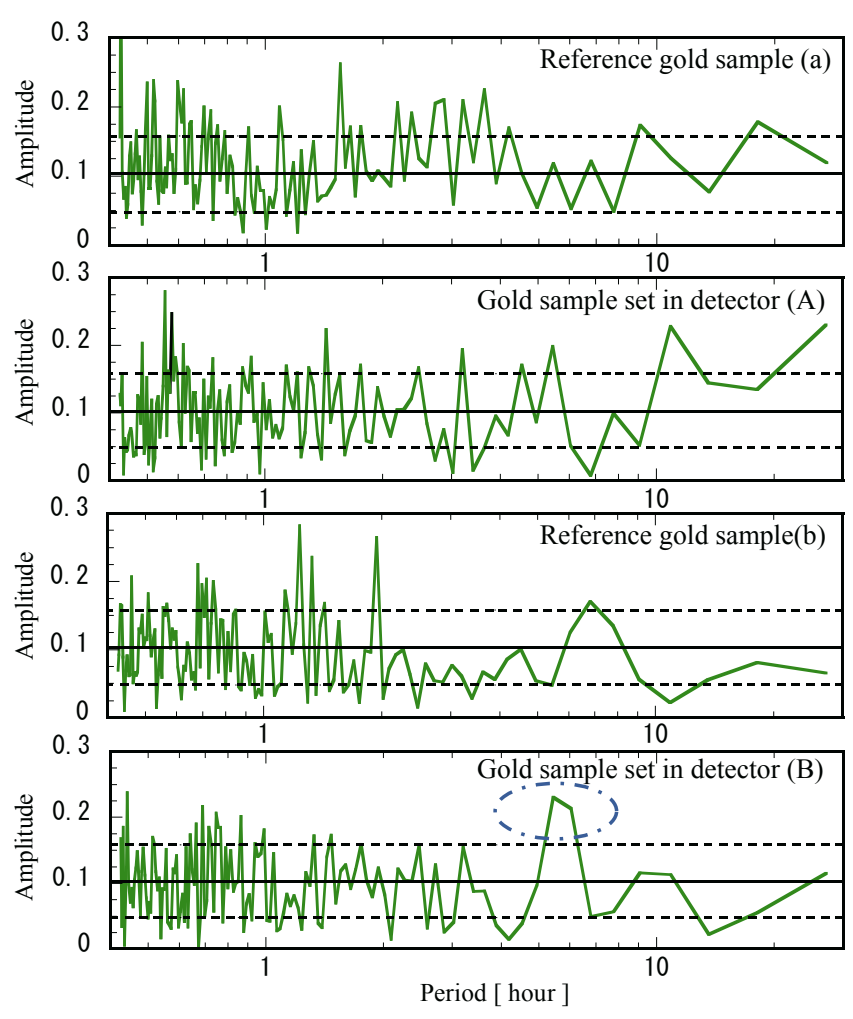

Fig. 3 Amplitudes of FFT results for correlativity $Y$. Dashed lines indicate $1 \sigma: 0.101 \pm 0.054$.

Fourier Transformation (DFT) and decreases the amount of operations, leading to high speed. For all experimental data, $Y$ values of 256 data were analyzed by FFT. Amplitudes of four FFT results for correlativity $Y$ are shown in Fig. 3. The amplitude of FFT result indicates the absolute value for sine and cosine components. Since the FFT periodicity results are symmetric, only the half area is displayed (128 data). The dash lines indicate the calculated result of one $\sigma$ in the ideal Poisson distribution case $(0.101 \pm 0.054)$ : the amplitude of period component for $Y$ scattered around zero with the standard deviation of $\sigma$. In all four cases, FFT results scatter by component by component. Almost all large components have small values in nearest adjacent points in Fig. 3 . The two adjacent components around a periodicity of 6 hours in (B) gave significantly large values.

The mean values of two adjacent components were obtained and are shown in Fig. 4. The dash lines indicate one $\sigma$ in the ideal case $(0.101 \pm 0.037)$ again. Three- $\sigma$ deviation indicates the reliability $95.4 \%$. The results on (B) showed that the component around 6 hours gave a clear deviation from the case of Poisson distribution. The deviation slightly exceeded three $\sigma$ of standard variation. A simple statistical estimation gives this probability below $1 \%$. Further experiment may be required for clearly concluding that it came from the existence of scalar auxiliary field $B^{0}$.

\section{Conclusion}

The radioisotope ${ }^{198} \mathrm{Au}$ was produced in gold foils $(10 \mathrm{~mm}$ $\times 10 \mathrm{~mm} \times 0.5 \mathrm{~mm})$ by ${ }^{197} \mathrm{Au}\left(\mathrm{n}_{\text {th }}, \gamma\right){ }^{198} \mathrm{Au}$ reaction in a thermal neutron flux of $10^{7}\left[\mathrm{~cm}^{-2} \cdot \mathrm{s}^{-1}\right]$ for 2 hours at Kinki 

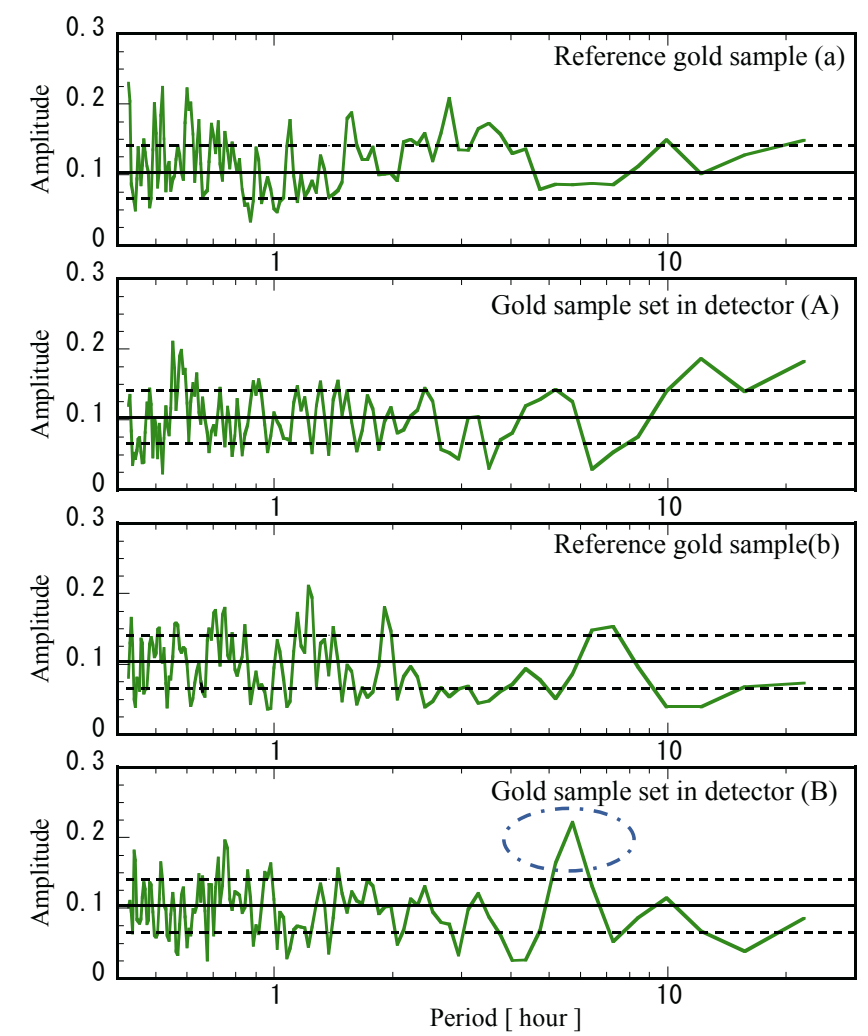

Fig. 4 Four kinds of calculated data of $256 Y$ values using FFT. Dashed lines indicate $1 \sigma .0 .101 \pm$ 0.037 .

University Atomic Energy Research Institute. Gamma rays were measured by the high-purity Ge detector for the activated gold samples as reference data. The experiments were carried out for electrochemical detectors with output voltage of 60 and $135 \mathrm{mV}$. The other activated gold samples were placed in the purified water of the electrochemical neutrino detector. The gamma-photo peak area at $0.412-\mathrm{MeV}$ was obtained for individual 15 -second measured data. The decrease rate of measured gamma-ray counts indicated that the half-life was unchanged.
The correlativity $Y$ was analyzed by FFT with 256 data. The results on the detector of $135 \mathrm{mV}$ showed that the component with periodicity of 6 hours gave statistical meaningful deviation of $99 \%$ confidence from the case of Poisson distribution. Further experiment may be required for concluding that it came from the existence of scalar auxiliary field $B^{0}$.

\section{Acknowledgment}

Authors express our gratitude to Prof. Tetsuo Horiguchi and Prof. Hiroshige Morishima of Kinki University Atomic Energy Research Institute for making radioisotope ${ }^{198} \mathrm{Au}$ and measuring gamma rays.

\section{References}

1) H. W. Johlige, D. C. Aumann and H. J. Born, "Detection of the Relative Electron Density at the Be Nucleus in Different Chemical Combination, Measured as Changes in the Electron-Capture Half-Life of ${ }^{7}$ Be," Phys. Rev. C2, 1616 (1970).

2) T. Nishimura, K. Ishibashi, et al., "Proposed structure of Neutrino Based on Weak-Charge and Weak-Dipole-Moment Interaction," Memoirs of the Faculty of Engineering, Kyushu University, 68, No.1, 61 (2008).

3) Liu Wei, K. Ishibashi, et al., "Possible detection of natural neutrinos by use of small apparatus," J. Nucl. Sci. Technol., Suppl. 4, 487 (2004).

4) K. Ishibashi, Liu Wei, et al., "Electrochemical neutrino detector and its signal generation scenario in relation to oxygen production," presented at this symposium.

5) K. Ishibashi, H. Arima, et al., "Observation of fluctuation in beta-decay of ${ }^{198} \mathrm{Au}$ placed in a small apparatus for neutrino detection," Annual Report of Kinki University Atomic Energy Research Institute (in Japanese), 40, 1 (2003). 